Laura Sciacovelli, Ada Aita, Andrea Padoan, Michela Pelloso, Giorgia Antonelli, Elisa Piva, Maria Laura Chiozza and Mario Plebani*

\title{
Performance criteria and quality indicators for the post-analytical phase
}

DOI 10.1515/cclm-2015-0897

Received September 14, 2015; accepted November 1, 2015; previously published online December 10, 2015

\begin{abstract}
Background: Quality indicators (QIs) used as performance measurements are an effective tool in accurately estimating quality, identifying problems that may need to be addressed, and monitoring the processes over time. In Laboratory Medicine, QIs should cover all steps of the testing process, as error studies have confirmed that most errors occur in the pre- and post-analytical phase of testing. Aim of the present study is to provide preliminary results on QIs and related performance criteria in the postanalytical phase.
\end{abstract}

Methods: This work was conducted according to a previously described study design based on the voluntary participation of clinical laboratories in the project on QIs of the Working Group "Laboratory Errors and Patient Safety” (WG-LEPS) of the International Federation of Clinical Chemistry and Laboratory Medicine (IFCC).

Results: Overall, data collected highlighted an improvement or stability in performances over time for all reported indicators thus demonstrating that the use of QIs is effective in the quality improvement strategy. Moreover, QIs data are an important source for defining the stateof-the-art concerning the error rate in the total testing process. The definition of performance specifications based on the state-of-the-art, as suggested by consensus documents, is a valuable benchmark point in evaluating the performance of each laboratory.

Conclusions: Laboratory tests play a relevant role in the monitoring and evaluation of the efficacy of patient

*Corresponding author: Mario Plebani, Department of Laboratory Medicine, University-Hospital of Padua, Via Giustiniani, 2, 35128 Padua, Italy, Phone: +39 0498212792, Fax: +39 049663240 ,

E-mail: mario.plebani@unipd.it.

http://orcid.org/0000-0002-0270-1711

Laura Sciacovelli, Ada Aita, Andrea Padoan, Michela Pelloso, Giorgia Antonelli and Elisa Piva: Department of Laboratory Medicine, University-Hospital of Padua, Italy

Maria Laura Chiozza: Department of Quality and Accreditation, University-Hospital of Padua, Padua, Italy outcome thus assisting clinicians in decision-making. Laboratory performance evaluation is therefore crucial to providing patients with safe, effective and efficient care.

Keywords: performance criteria; post-analytical phase; quality indicators.

\section{Introduction}

Efforts to reduce errors and enhance patient safety in medicine must focus on risk procedures and processes with a high potential for error generation. Achieving consistently high levels of quality in laboratory medicine calls for moving beyond analytic activities. In the last few decades, performance measurements have focused mostly on the analytical processes with a view to meeting the quality specifications of precision and trueness [1, 2]. Internal Quality Control (IQC) procedures and External Quality Assessment Programs (EQA) have significantly improved the intra-analytical quality of laboratory testing; however, medical error studies confirm that most errors occur in the pre- and post-analytical phases of testing [3-6]. The implementation of performance measurements to evaluate the pre- and post-analytical stages of the total testing process (TTP) is therefore needed in order to maximize the overall testing cycle and the quality of patient care. In addition, recent regulation and accreditation guidelines now require laboratories to focus their improvement efforts on, not only on the analytical step, but also the other steps of the TTP [7]. Performance measurement, an important component of the quality management system, has been a core feature of quality improvement programs across many health organizations. In laboratory medicine this valuable tool can improve and standardize performance and, by reducing inefficiency, can lead to a better health outcome.

The use of quality indicators (QIs), as performance measurements, is an effective tool for achieving an accurate estimate of the degree of quality, identifying problems that may need to be addressed, and monitoring processes over time [8]. A project of the Working Group "Laboratory 
Errors and Patient Safety" (WG-LEPS) of the International Federation of Clinical Chemistry and Laboratory Medicine (IFCC) has provided a list of QIs (MQI) for monitoring all TTP activities. This project has been designed to define appropriate QIs for use in all laboratories worldwide, collect data from them, identify the current state-of-the-art and define performance criteria to better address improvement actions, decrease the error rate and suggest steps to take in order to further improve performance [9-11].

Aim of the present study was to present the state-ofthe-art concerning QIs for the post-analytical phase on the basis of the consensually defined MQI, and submit a proposal for identification of related performance specifications. The list of consensually defined QIs, and the rationale and aims of the Project, described elsewhere [11], are available on a specifically developed website (www.ifcc-mqi.com). The rationale of the selected QIs in the post-analytical phase is briefly summarized below.

\section{Errors and quality indicators in the post-analytical phase}

Poor communication between laboratory professionals and clinicians is generally cited as a chief issue affecting quality during the pre- and post-analytical phases. In particular, post-analytic communication entails laboratory professionals' communications with the clinician about timeliness of reporting, notification of significant abnormal test results, and presentation of relevant information through reports and interpretative comments. Breakdowns in communication lead to errors, events affecting patient safety, and inefficient and ineffective use of resources.

QIs, an effective tool for monitoring all activities in TTP, have been well described in several studies $[8,12]$, but laboratories have highlighted the difficulties involved in using them, particularly in the post-analytical phase, when for data collection calls for the clinicians' collaboration.

\section{Turnaround time (TAT)}

Clinicians are interested in service quality, which encompasses total testing error (imprecision and trueness), availability, cost, relevance and timeliness. However, since they often judge the quality of a laboratory on the basis of timeliness, many laboratories may be ready to sacrifice analytical quality for a faster TAT.
The timeliness with which test results are delivered is one of the most prominent parameters of laboratory medicine and a common indicator of performance, which is measured by monitoring the TAT of some specific tests, and the time for notification of critical results. The automation of various steps in the analytical phase, the increased use of electronic results reporting, and the development of automatic electronic alerting systems for critical values have contributed to reducing the time of results reporting. Prompt reporting of test results can improve efficiency in patient care and enhance clinician and patient satisfaction, even when it does not affect health outcomes [13].

The correct monitoring of times calls for the knowledge of the different measurement approaches used by laboratories. For example, test typology, need for priority reporting, (e.g. urgent or routine), patient typology (e.g. inpatients, outpatients, urgent cases) and the included activities (interval of measurement). The different factors characterizing TAT measurement compromise comparability of data among laboratories. Typically, TAT is assessed by determining the difference between recorded starting times (test ordering or specimen collection time or laboratory sample receipt time) and end points (test reporting time). Some laboratories are also expanding the scope of measurement by evaluating "therapeutic TAT", the time from test order initiation to clinical decision implementation (e.g. change in treatment) [14].

The choice of timing points (start and end) is relevant to identifying the state-of-the-art and defining related quality specifications. The most widely used TAT measurement includes the activities under laboratory control, from sample receipt to the result sending. The choice of statistical approach for data analysis must also be defined to allow reliable evaluation of data over time. For example, the mean and standard deviation are not an appropriate bases for defining TAT distribution, the most commonly used measurement being the time interval during which $90 \%$ of results are completed (corresponding to 90th percentile) [15].

The use of QIs, which have well defined measurement characteristics, is therefore important in monitoring the TAT, knowing the state-of-the-art and identifying the improvement possibilities. Because TAT monitoring for all tests provided by the laboratory would require a lot of work, only some test have been chosen in the MQI as representative of laboratory performance in relation to timing. Moreover, to enhance comparability between participants' data, TAT was measured only on urgent samples (shortest turnaround time priority, STAT) in which it is probably affected less by differences in the inherent features of laboratories (e.g. different size and workload). 


\section{Notification of critical values}

Critical values notification procedures play a key role in safe and effective patient care. To assure effective procedures, every clinical laboratory must periodically review its list of critical values, both for internal policy and for clinical purpose, and review their procedures [16]. The continuous monitoring of successfully communicated critical values within a defined time and relative time measurement helps laboratories to focus with renewed vigor on critical value management. Yet there is still a lack of consensus and benchmark data. Nor has harmonization been achieved for procedures, choice of analytes and critical ranges and moreover, notification times vary depending on patient typology (inpatients or outpatients). Systematic monitoring using consensually approved QIs helps laboratories to evaluate their performances, and to compare them with that of other laboratories.

\section{Laboratory reports}

Errors leading to incorrect or delayed patient results can affect medical decisions and compromise the efficacy of patient treatment. The content, format and physical presentation of information provided can significantly affect the interpretation and use of laboratory data by clinicians. Mistakes in the content and completeness of laboratory reports as well as misunderstanding by the treating physician as to the significance of the information in the report, among other factors, can delay the treatment of a serious disease and alter outcomes. Specific report content issues can include any of the following: uninterpretable information, incorrect data of reference intervals, inaccurate personal details of patient or incorrect reporting of measurand. Moreover, different types of error can occur during report formatting. Reports that lack units of measurement or use inappropriate units of measurement can lead to harmful misinterpretation of results and/or undervaluation of important information. The definition of QIs that includes the measurement of these aspects aims to obviate any misinterpretation and to promote accuracy and completeness.

\section{Interpretative comments with a positive impact on the patient outcome}

The aim of interpretative comments on laboratory reports is to help clinicians to interpret complex data provided, particularly when dynamic or uncommon test results are reported, when significant abnormalities are present, and/or when analytical or pre-analytical factors may compromise the interpretation of results. Although several authors have described this process and indicated its value, there is little evidence that it has improved patient outcomes, mainly due to difficulties involved in collecting data [17-20]. The interpretation of results is crucial to patient outcome yet, hoping to avoid giving inappropriate advice, many laboratories fail to provide interpretative comments in the absence of complete clinical information. According to studies available in the literature, the majority of comments provided in laboratory reports are acceptable, but some are inappropriate or misleading and, in a few cases, dangerous, leading to inaccurate assumptions by staff, especially if the available clinical information is insufficient or expertise in a clinical chemistry subspecialty area (e.g. toxicology, endocrinology, and tumor markers) is inadequate [21].

Interpretative comments in line with current guidelines are generally welcomed by physicians because they prevent an inordinate focus on certain values from leading to a disregard of other potentially important values [22, 23]. The use of QIs to monitor the effectiveness of interpretative comments provided should therefore be encouraged and developed because a laboratory result is not complete until it has been interpreted and incorporated into patient care, as appropriate.

\section{Materials and methods}

The present work was conducted according to a previously study design described elsewhere [12] and relied on the voluntary participation of clinical laboratories worldwide enrolled in the IFCC WG-LEPS project on QIs [11]. Two hundred and eighty-one laboratories are enrolled in the project from different countries (Table 1) but the number of participating laboratories changed over time. In addition, the number may differ from a indicator to another one as any clinical laboratory may select some QIs and is not obliged to collect data for the entire list. Thirty-nine clinical laboratories (2 Argentina, 1 Brazil, 2 China, 1 Croatia, 1 Estonia, 1 Great Britain, 1 Ireland, 12 Italy, 16 Serbia, 1 Spain, 1 Uruguay) continuously and systematically collected data for QIs of the post-analytical phase in the timeframe considered in the present study (from 2012 to 2014). Public laboratories account for $82 \%$ of participants while $18 \%$ are private Institutions.

Data collection was undertaken quarterly for all QIs evaluated except for TAT, for which it was performed monthly.

The results have been reported in a percentage (\%) and, in addition where appropriate, the short term sigma has been calculated for each result as the Six Sigma metric is widely recognized as "a metric for measuring defects and improving quality" [24, 25]. 
Table 1: Laboratories involved in the project on MQI of the IFCC WG-LEPS.

\begin{tabular}{lrlr}
\hline Countries & Labs, $\mathbf{n}$ & Countries & Labs, $\mathbf{n}$ \\
\hline Algeria & 1 & Italy & 74 \\
Argentina & 13 & Libya & 1 \\
Australia & 4 & Netherlands & 2 \\
Austria & 2 & New Zealand & 1 \\
Belgium & 5 & Palestine & 1 \\
Brazil & 12 & Poland & 1 \\
Canada & 8 & Portugal & 3 \\
China & 45 & Saudi Arabia & 2 \\
Croatia & 6 & Serbia & 25 \\
Egypt & 1 & Singapore & 2 \\
Estonia & 3 & Slovenia & 4 \\
France & 1 & Spain & 11 \\
Germany & 7 & Sweden & 3 \\
Great Britain & 13 & Switzerland & 2 \\
Greece & 2 & Turkey & 5 \\
Hungary & 2 & United Arab Emirates & 2 \\
India & 4 & United States & 7 \\
Ireland & 3 & Uruguay & 2 \\
Israel & 1 & & \\
\hline
\end{tabular}

\section{Results}

Table 2 shows participants' results for the post-analytical phase, collected in the years 2012, 2013 and 2014, and expressed as a 50th percentile/median. Overall, performance for all indicators was stable or had improved. The data collected in the different years (2012, 2013, 2014) are surely affected by different typologies and number of laboratories; however this should be not considered a limitation when evaluating the changes over time (improvement or worsening).

\section{TAT}

QIs data for TAT concerning the most urgent priority samples (STAT) are reported in Table 2: Potassium, from 2012 to 2014; International Normalized Ratio (INR), white blood cell count (WBC) and troponin only for 2014 because these values were added during the Consensus Conference on QIs held in 2013 [10]. Potassium TAT highlights a variable trend from 2012 to 2014 and a wide range

Table 2: Median value calculated on all results provided by laboratories participating in the IFCC "Laboratory Errors and Patient Safety" project on quality indicators, from 2012 to 2014.

\begin{tabular}{|c|c|c|c|}
\hline Quality indicators for the post-analytical phase & Unit & Year & Median \\
\hline \multicolumn{4}{|l|}{ Turnaround times } \\
\hline \multirow[t]{3}{*}{ Number of reports delivered outside the specified time/total number of reports } & Percentage & 2012 & 0.66 \\
\hline & & 2013 & 0.67 \\
\hline & & 2014 & 0.060 \\
\hline \multirow[t]{3}{*}{ Turnaround time (min) of potassium (K) at 90th percentile (STAT) } & Time & 2012 & 59.0 \\
\hline & & 2013 & 48.0 \\
\hline & & 2014 & 53.2 \\
\hline Turnaround time (min) of International normalized ratio (INR) value at 90th percentile (STAT) & Time & 2014 & 54 \\
\hline Turnaround time (min) of white blood cell count (WBC) at 90th percentile (STAT) & Time & 2014 & 28 \\
\hline Turnaround time $(\mathrm{min})$ of troponin I $(\mathrm{TnI})$ or troponin $\mathrm{T}(\mathrm{TnT})$ at 90th percentile (STAT) & Time & 2014 & 68.82 \\
\hline \multicolumn{4}{|l|}{ Laboratory reports } \\
\hline \multirow{3}{*}{$\begin{array}{l}\text { Number of incorrect reports issued by the laboratory/total number of reports issued by the } \\
\text { laboratory }\end{array}$} & Percentage & 2012 & 0.009 \\
\hline & & 2013 & 0.009 \\
\hline & & 2014 & 0.008 \\
\hline \multicolumn{4}{|l|}{ Notification of critical values } \\
\hline \multirow{3}{*}{$\begin{array}{l}\text { Number of critical values notified after a consensually agreed time (from result validation to } \\
\text { result communication to the clinician)/total number of critical values to communicate }\end{array}$} & Percentage & 2012 & 67.2 \\
\hline & & 2013 & 17.8 \\
\hline & & 2014 & 0 \\
\hline $\begin{array}{l}\text { Time taken (from result validation to result communication to clinician) to communicate } \\
\text { critical values of inpatients }(\mathrm{min})\end{array}$ & Time & 2014 & 4.66 \\
\hline $\begin{array}{l}\text { Time taken (from result validation to result communication to clinician) to communicate } \\
\text { critical values of outpatient (min) }\end{array}$ & Time & 2014 & 11.03 \\
\hline
\end{tabular}




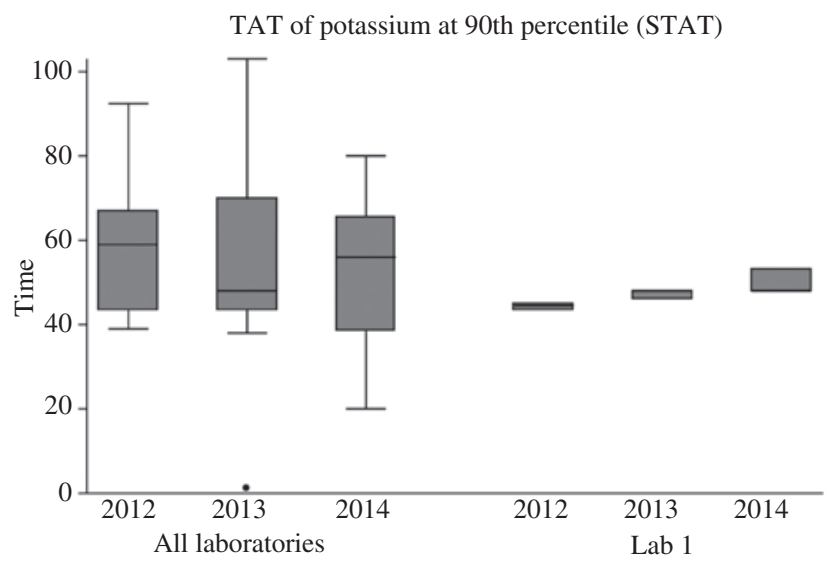

Figure 1: Turnaround time (TAT) of potassium in emergency: graph showing statistical measures (median, upper and lower quartiles, the minimum and maximum of all data and outliers).

of results within 1 year (Figure 1), underlining the different results from participating laboratories. As expected, the variability of results from a single laboratory (Lab. 1) is narrower.

\section{Notification of critical values}

The QIs proposed by IFCC WG-LEPS for critical values aim to determine the level of successful reporting of critical values in the laboratory, for inpatients and outpatients, and to ensure that participant results are comparable across institutions by providing a clear definition of time measurement (start and end points). When there was evidence that the critical value was communicated according to a given laboratory's policy and procedure, the notification was considered successful. The data collected highlight that the percentage of critical values notified after the defined time significantly decreased over time for all laboratories (Kruskal-Wallis rank test: $\left.\mathrm{X}^{2}=16.9, \mathrm{p}<0.001\right)$ and Lab. 1 (Kruskal-Wallis rank test: $\left.\mathrm{X}^{2}=5.33, \mathrm{p}=0.021\right)$ demonstrating that Laboratories have developed practices ensuring that each critical result is promptly transmitted to caregivers, and that staff awareness of this issue has been raised. The monitoring of notification time highlights the fact that procedures are carried out rapidly and effectively (Table 2 and Figure 2).

The time needed to communicate outpatients' results was found to be longer than that required for communicating inpatients' results (median time, $11.03 \mathrm{~min}$ and $4.66 \mathrm{~min}$, respectively).

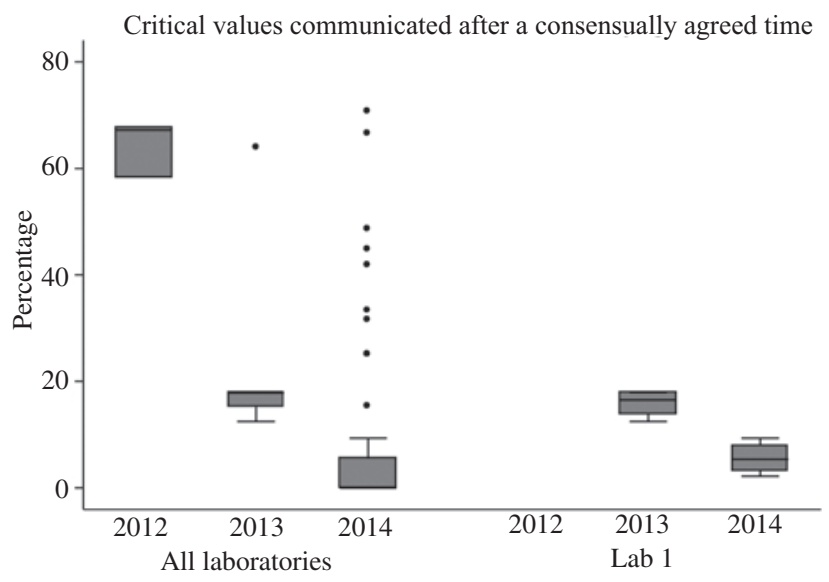

Figure 2: Critical values notification: graph showing statistical measures (median, upper and lower quartiles, the minimum and maximum of all data and outliers).

\section{Laboratory reports}

Data demonstrated a decrease in the number of reports delivered outside the specified time during the year (Table 2), and an improvement in quality level (short term sigma, from 3.9 to 4.5). Whereas a stable pattern for incorrect laboratory reports is highlighted (Table 2) with a high quality level for process (short-term sigma, from 5.1 to 5.2).

\section{Interpretative comments with a positive impact on the patient outcome}

The laboratories did not collect data on QIs for interpretative comments; the explanation for this is given in the discussion section.

\section{Performance specifications}

Table 3 reports on the proposal for performance specifications drawn up on the basis of data collected in 2014 in order to provide a reliable picture of the current stateof-the-art, and in according with the proposal by Fraser et al. [26] that classified the performances into: optimum, desirable, and minimum [12]. However, as the ideal performance criteria should be "zero defect", we made a preliminary definition of the following three levels: high, medium and low. In fact, while for analytical performance criteria the levels are defined with respect to biological variation, for pre- and post-analytical issues, errors and defects are linked specifically to the quality of the 
Table 3: Proposal for quality specifications based on percentile values calculated on the basis of laboratories' results (expressed as percentages and, where appropriate, short term sigma) collected in 2014.

\begin{tabular}{|c|c|c|c|c|}
\hline \multirow[t]{2}{*}{ Quality indicators } & \multirow[t]{2}{*}{ Unit } & \multicolumn{3}{|c|}{$\begin{array}{l}\text { Performance specifications based } \\
\text { on } 75 \text { th }-50 \text { th }-25 \text { th percentile }\end{array}$} \\
\hline & & Low & Medium & High \\
\hline \multirow[t]{2}{*}{ Number of reports delivered outside the specified time/total number of reports } & Percentage & 0.22 & 0.060 & 0.006 \\
\hline & Sigma & 3.904 & 4.5 & 4.795 \\
\hline Turnaround time (min) of potassium (K) at 90th percentile (STAT) & Time & 66 & 53.2 & 39 \\
\hline Number of incorrect reports issued by the laboratory/total number of reports & Percentage & 0.035 & 0.008 & 0.0002 \\
\hline issued by the laboratory & Sigma & 4.7 & 5.1 & 5.4 \\
\hline $\begin{array}{l}\text { Number of critical values of inpatients notified after a consensually agreed time } \\
\text { (from result validation to result communication to the clinician)/total number of } \\
\text { critical values of inpatients to communicate }\end{array}$ & Percentage & 6.62 & $2.43^{\mathrm{a}}$ & 0 \\
\hline $\begin{array}{l}\text { Number of critical values of outpatients notified after a consensually agreed time } \\
\text { (from result validation to result communication to the clinician)/total number of } \\
\text { critical values of outpatients to communicate }\end{array}$ & Percentage & 7.14 & $5.00^{\mathrm{a}}$ & 0 \\
\hline
\end{tabular}

${ }^{a}$ These values are different from those reported in Table 2 because, the median value is now calculated on data grouped by patient typology (inpatient and outpatient) on the basis of the improved formulation of the indicator introduced in 2014.

procedures and, at least in theory, the final goal is "zero tolerance". This approach allows laboratories not only to ascertain whether their performance lies within an acceptable range, but also to recognize a possible negative trend when their performance shifts from a high, to a medium or low level (e.g. Figure 1, Lab. 1).

\section{Discussion}

The activities in the post-analytical phase call for a sound understanding of the analytical processes involved in generating results, the awareness of potential preanalytical and intra-analytical errors, and the correlation of results with the patient's clinical status. The collaboration between pathologist and clinician is of fundamental importance in ensuring that this process is effective.

The knowledge of state-of-the-art is the simplest possible means for enabling laboratories to reliably compare their performances. It has been accepted as a basis for defining specifications for analytical quality in the hierarchical model described in the Stockholm Consensus Conference [1], reviewed in the Milan 1st EFLM Strategic Conference [2], and used in the present study to define specifications for post-analytical quality.

The use of QIs for post-analytical phases, although still not widely adopted [27], is important for assessing the error rate and determining the level of risk acceptable for prioritizing interventions, and is required by the
International Standard for laboratory accreditation (ISO 15189:2012) [7]. The performance specifications define the extent of change in performance to be allowed before the specific activity under control can be considered out-of-control.

Participation in inter-laboratory comparison on QIs, such as that proposed by IFCC WG-LEPS, raises awareness of the significance of both quality-enhancing practice and the current level of performance. Moreover, continuous QIs monitoring is helpful in the provision of education and continuing professional development, and contributes to the improvement of patient care and minimization of medical errors.

The data collected show that the variability observed in the TAT results of all laboratories can depend on the different policies and procedures used by the individual laboratories. Shorter TAT measurements do not necessarily indicate a superior performance, as they do not reflect whether the laboratory concerned meets the expectations of clinicians using its services. It is known, however, that the majority of identified problems directly affecting TAT (e.g. for selected chemistry tests) are associated with preanalytical steps (e.g. test ordering and sample collection) and analytical-related personnel and technical issues. The use of QIs allows the identification of appropriate improving actions taken as a result of regular monitoring, and may therefore improve laboratory performance $[28,29]$. Regarding laboratory reports, the feed-back from participating laboratories in the MQI project of the IFCC WG-LEPS highlighted that the collection of these data 
is straightforward and, although there may be room for improvement, the process is under control.

The time taken to communicate critical values to outpatients is longer than that for inpatients because the notification procedure is often difficult in the former case, as laboratory professionals may make repeated attempts to contact the appropriate outpatient's physician, sometimes unsuccessfully. The communication process for inpatients is easier due to the constant presence of physicians in clinical wards. Moreover, the communication method can affect the notification time: most institutions rely on the telephone, while others use electronic devices that automatically dial a beeper or send a short message service (SMS), thus reducing the time spent in critical value notification - and increasing the likelihood that the physician, or another person in charge of the patient's care, will receive the result [30, 31]. Further QIs could be established for monitoring critical values procedures and promoting harmonization. For example, close attention must be paid to the following: staff receiving critical values notification (the person receiving the information must be authorized and capable of interpreting and using the results appropriately); the procedure used when critical values from the same patients are observed over the following days; the procedure used during the notification; the clinical wards to exclude from receiving the notification because all patients are critical; the list of analytes; the lower and higher limit values. The evidence of many outliers, particularly in 2014 (see Figure 2), that led to unsatisfactory performances highlights, once again, the need for a careful revision of internal policies and procedures to achieve better performances. This, in turn, reinforces the use of the data for a valuable benchmark and harmonization between different clinical laboratories.

Findings in an investigation into the reasons for not having measured the effectiveness of interpretative comments highlight that it is both difficult and time-consuming to collaborate with the clinician in order to evaluate an outcome following the introduction of a specific interpretative comment in the patient's report. Moreover, physicians often fail to record the actions undertaken in response to laboratory reports. The need to evaluate and monitor outcome measures in order to assure the effectiveness of laboratory processes calls for an improvement in the communication between clinicians and laboratory professionals in the ordering of tests and the interpretation of results [32]. The paper presents some limitations and, in particular, the number of data collected by a limited number of clinical laboratories requires further confirmation in future surveys.

\section{Conclusions}

In Laboratory Medicine, the time is now ripe to focus attention on the assessment and monitoring procedures for the extra-analytical quality. Professionals are responsible for facilitating the assimilation and comprehension of laboratory information by clinicians, and efforts should be made to identify the areas in which the harmonized use of consensually defined QIs is needed, in all laboratories worldwide, in order to strive for improvement in intraanalytical activities by means of the continuous use of IQC and EQA, also in the extra-analytical phases. Further efforts must be made to encourage laboratories to collect QIs data and undertake the actions for improvement when results go beyond the defined quality specifications.

Since laboratory tests play an extremely important role in monitoring and evaluating patient outcomes and assisting clinicians in their decision making, the rigorous evaluation of laboratory performance is crucial to providing patients with safe, effective and efficient care.

Author contributions: All the authors have accepted responsibility for the entire content of this submitted manuscript and approved submission.

Research funding: None declared.

Employment or leadership: None declared.

Honorarium: None declared.

Competing interests: The funding organization(s) played no role in the study design; in the collection, analysis, and interpretation of data; in the writing of the report; or in the decision to submit the report for publication.

\section{References}

1. Kallner A, McQueen M, Heuck C. The Stockholm Consensus Conference on quality specifications in laboratory medicine, 25-26 April 1999. Scand J Clin Lab Invest 1999;59:475-6.

2. Sandberg S, Fraser CG, Horvath AR, Jansen R, Jones G, Oosterhuis W, et al. Defining analytical performance specifications: Consensus Statement from the $1^{\text {st }}$ Strategic Conference of the European Federation of Clinical Chemistry and Laboratory Medicine. Clin Chem Lab Med 2015;53:833-5.

3. Bonini P, Plebani M, Ceriotti F, Rubboli F. Errors in laboratory medicine. Clin Chem 2002;48:691-8.

4. Plebani $M$. The detection and prevention of errors in laboratory medicine. Ann Clin Biochem 2010;47:101-10.

5. Plebani M, Carraro P. Mistakes in a stat laboratory: types and frequency. Clin Chem 1997;43:1348-513.

6. Carraro P, Plebani M. Errors in a stat laboratory: types and frequencies 10 years later. Clin Chem 2007;53:1338-42.

7. UNI EN ISO 15189:2013. Medical laboratories - Requirements for quality and competence. Geneva, Switzerland: International Organization for Standardization, 2013. 
8. Institute of Medicine. To err is human: building a safer health system. Washington, DC: National Academy Press, 2000.

9. Sciacovelli L, Plebani M. The IFCC Working Group on laboratory errors and patient safety. Clin Chim Acta 2009;404:79-85.

10. Sciacovelli L, O’Kane M, Skaik YA, Caciagli P, Pellegrini C, Da Rin G, et al. IFCC WG-LEPS. Quality Indicators in Laboratory Medicine: from theory to practice. Preliminary data from the IFCC Working Group Project "Laboratory Errors and Patient Safety”. Clin Chem Lab Med 2011;49:835-44.

11. Plebani M, Astion ML, Barth JH, Chen W, de Oliveira Galoro CA, Escuer MI, et al. Harmonization of quality indicators in laboratory medicine. A preliminary consensus. Clin Chem Lab Med 2014;52:951-8.

12. Plebani M, Sciacovelli L, Aita A, Pelloso M, Chiozza ML. Performance criteria and quality indicators for the pre-analytical phase. Clin Chem Lab Med 2015;53:943-8.

13. Valestein P. Laboratory turnaround time. Am J Clin Pathol 1196;105:676-88.

14. Kilgore ML, Steindel SJ, Smith JA. Evaluating stat testing options in an academic health center: therapeutic turnaround time and staff satisfaction. Clin Chem 1998;44:1597-603.

15. Hawkins RC. Laboratory turnaround time. Clin Biochem Rev 2007;28:179-1949.

16. Piva E, Sciacovelli L, Laposata M, Plebani M. Assessment of critical values policies in Italian institutions: comparison with the US situation. Clin Chem Lab Med 2010;48:461-8.

17. Dighe AS, Sodeberg BI, Laposata M. Narrative interpretation for clinical laboratory interpretations. Am J Clin Pathol 2001;116:S123-8.

18. Macmillian DH, Sodeberg BI, Laposata M. Regulations regarding reflexive testing and narrative interpretations in laboratory medicine. Am J Clin Pathol 2001;116:S129-32.

19. Kratz A, Sodeberg BI. The generation of narrative interpretations in laboratory medicine. Am J Clin Pathol 2001;116:S133-40.

20. Plebani M. Interpretative commenting: a tool for improving the laboratory-clinical interface. Clin Chim Acta 2009;404:46-51.
21. Lim EM, Sikaris KA, Gill J, Calleja J, Hickman PE, Beilby J, et al. Quality assessment of interpretative commenting in clinical chemistry. Clin Chem 2004;50:632-7.

22. Laposata ME, Laposata M, Van Cott EM, Buchner DS, Kashalo MS, Dighe AS. Physician survey of a laboratory medicine interpretive service and evaluation of the influence of interpretations on laboratory test ordering. Arch Pathol Lab Med 2004;128:1424-7.

23. Zarbo RJ, Nakhleh RE, Walsh M. Customer satisfaction in anatomic pathology: a College of American Pathologists Q-Probes study of 30656 physician surveys from 94 laboratories. Arch Pathol Lab Med 2003;127:23-9.

24. Westgard JO. Six sigma quality, design and control. Madison, WI: Westgard QC, 2006.

25. Burnett D. A practical guide to ISO 15189 in Laboratory medicine. London: Association for Clinical Biochemistry and Laboratory Medicine, 2013.

26. Fraser CG, Hylton Petersen P, Libeer J-C, Ricos C. Proposal for setting generally applicable quality goals solely based on biology. Ann Clin Biochem 1997;34:1-8.

27. Ricos C, Garcia-Victoria M, de la Fuente B. Quality indicators and specifications for the extra-analytical phases in clinical laboratory management. Clin Chem Lab Med 2004;42:578-82.

28. Novis DA, Walsh MK, Dale JC, Howanitz PJ. Continuous monitoring of stat and routine outlier turnaround times: two College of American Pathologists Q-Track monitors in 2941 hospitals. Arch Pathol Lab Med 2004;128:621-6.

29. Steindel SJ, Novis DA. Using outlier event to monitor test turnaround time: a College of American Pathologists Q-Probes study in 496 laboratories. Arch Pathol Lab Med 1999;123:607-14.

30. Piva E, Pelloso M, Penello L, Plebani M. Laboratory critical values: automated notification supports effective clinical decision making. Clin Biochem 2014;47:1163-8.

31. Piva E, Sciacovelli L, Zaninotto M, Laposata M, Plebani M. Evaluation of effectiveness of a computerized notification system for reporting critical values. Am J Clin Pathol 2009;131:432-41.

32. Sikaris K. Performance criteria of the post-analytical phase. Clin Chem Lab Med 2015;35:949-58. 